\title{
SUM OF A DOUBLE SERIES
}

\section{B. L. SHARMA}

ABSTRACT. In this paper we obtain the sum of a double series $F(1,1)$ and, in a particular case, we get a new formula ${ }_{4} F_{3}(1)$,

$$
{ }_{4} F_{3}\left[\begin{array}{c}
\alpha, \beta-\alpha, 1 / 2 \rho, 1 / 2 \rho+1 / 2 ; 1 \\
1 / 2 \beta, 1 / 2(1+\beta), 1+\rho ;
\end{array}\right]=\frac{\Gamma(\beta-\rho-\alpha) \Gamma(\beta)}{\Gamma(\beta-\rho) \Gamma(\beta-a)},
$$

provided that $R(\beta-\alpha)>0, R(\beta-\rho-\alpha)>0$ and $R(\beta-\rho)>0$. If $a=-n$, the formula reduces to a known result due to Bailey [2].

1. Introduction. Recently, Professor Carlitz [4] proved a Saalschutzian theorem for a double series, and later [5] gave the sum of another double series。

Professor Carlitz' papers created interest in the summation formulae, because these formulae have applications in the solutions of certain problems in theoretical physics. Following Professor Carlitz' papers, Jain [6] and Sharma [8] have proved some new summation formulae for double series. The object of this paper is to give the sum of a certain double hypergeometric series, and on specializing the parameters, we get a result of Bailey [2, (8.2)]. The results obtained in this paper are believed to be new.

The following notation of Chaundy [3] will be used to represent the hypergeometric series of higher order and of two variables.

$$
F\left[\begin{array}{c}
\left(a_{p}\right) ;\left(b_{q}\right) ;\left(c_{r}\right) ; x, y \\
\left(d_{s}\right) ;\left(e_{h}\right) ;\left(f_{k}\right) ;
\end{array}\right]=\sum_{m, n=0}^{\infty} \frac{\left[\left(a_{p}\right)\right]_{m+n}\left[\left(b_{q}\right)\right]_{m}\left[\left(c_{r}\right)\right]_{n} x^{m} y^{n}}{\left[\left(d_{s+n}\left[\left(e_{h}\right)\right]_{m}\left[\left(f_{k}\right)\right]_{n} m ! n !\right.\right.},
$$

where $\left(a_{p}\right)$ and $\left[\left(a_{p}\right)\right]_{m+n}$ will mean $a_{1}, \ldots, a_{p}$ and $\left(a_{1}\right)_{m+n}, \ldots,\left(a_{p}\right)_{m+n}$.

In the investigation we require Slater's formula $[7$, p. $65,(2.4 .2 .2)]$ :

$$
{ }_{4} F_{3}\left[\begin{array}{c}
-n, 1+f-g, 1 / 2 f, 1 / 2 f+1 / 2 ; 1 \\
1+f, 1 / 2+1 / 2 f-1 / 2 g-1 / 2 n, 1+1 / 2 f-1 / 2 g-1 / 2 n ;
\end{array}\right]=\frac{(g)_{n}}{(g-f)_{n}} .
$$

2. The formula to be proved is

Received by the editors May 3, 1974 and, in revised form, December 18, 1974. AMS (MOS) subject classifications (1970). Primary 33A30.

Key words and phrases. Double series, Saalschutizian theorem, hypergeometric series of higher order and of two variables, summation formula. 


$$
\begin{gathered}
F\left[\begin{array}{c}
\alpha, \beta+f_{1}+f_{2}-\alpha ; 1 / 2 f_{1}, 1 / 2+1 / 2 f_{1} ; 1 / 2 f_{2}, 1 / 2+1 / 2 f_{2} ; 1,1 \\
1 / 2\left(\beta+f_{1}+f_{2}\right), 1 / 2\left(1+\beta+f_{1}+f_{2}\right) ; 1+f_{1} ; 1+f_{2} ;
\end{array}\right] \\
=\frac{\Gamma(\beta-\alpha) \Gamma\left(\beta+f_{1}+f_{2}\right)}{\Gamma(\beta) \Gamma\left(\beta+f_{1}+f_{2}-\alpha\right)},
\end{gathered}
$$

valid for $R(\beta)>0, R(\beta-\alpha)>0, R\left(\beta+f_{1}+f_{2}\right)>0$ and $R\left(\beta+f_{1}+f_{2}-\alpha\right)>0$.

Proof. To prove (3), we consider

$$
\begin{aligned}
\frac{\Gamma(\beta) \Gamma\left(\beta-g_{1}-g_{2}-\alpha\right)}{\Gamma(\beta-\alpha) \Gamma\left(\beta-g_{1}-g_{2}\right)}=\sum_{m=0}^{\infty} \sum_{n=0}^{\infty} \frac{(\alpha)_{m+n}\left(g_{1}\right)_{m}\left(g_{2}\right)_{n}}{(\beta)_{m+n} m ! n !} \\
=\sum_{m=0}^{\infty} \sum_{n=0}^{\infty} \frac{(\alpha)_{m+n}}{(\beta)_{m+n}} \sum_{r=0}^{m} \frac{(-m)_{r}\left(1+f_{1}-g_{1}\right)_{r}\left(1 / 2 f_{1}\right)_{r}\left(1 / 2+1 / 2 f_{1}\right)_{r}}{\left(1+f_{1}\right)_{r}\left(1 / 2+1 / 2 f_{1}-1 / 2 g_{1}-1 / 2 m\right)_{r}} \\
\cdot \frac{\left(g_{1}-f_{1}\right)_{m}}{\left(1+1 / 2 f_{1}-1 / 2 g_{1}-1 / 2 m\right)_{r} r} \sum_{s=0}^{n} \frac{(-n)_{s}\left(1+f_{2}-g_{2}\right)_{s}\left(1 / 2 f_{2}\right)_{s}}{\left(1+f_{2}\right)_{s}\left(1 / 2+1 / 2 f_{2}-1 / 2 g_{2}-1 / 2 n\right)_{s}} \\
\cdot \frac{\left(1 / 2+1 / 2 f_{2}\right)_{s}\left(g_{2}-f_{2}\right)_{s}}{\left(1+1 / 2 f_{2}-1 / 2 g_{2}-1 / 2 n\right)_{s} s !} \text { by }(2) \\
=\sum_{r=0}^{\infty} \sum_{s=0}^{\infty} \frac{(\alpha)_{r+s}\left(1 / 2 f_{1}\right)_{r}\left(1 / 2+1 / 2 f_{1}\right)_{r}\left(1 / 2 f_{2}\right)_{s}\left(1 / 2+1 / 2 f_{2}\right)_{s} 2^{2 r+2 s}}{\left(1+f_{1}\right)_{r}\left(1+f_{2}\right)_{s} r ! s !} \\
\cdot F_{1}\left[\alpha+r+s ; g_{1}-f_{1}-r, g_{2}-f_{2}-s ; \beta+r+s ; 1,1\right] .
\end{aligned}
$$

Now we make use of the formula of Appell and Kampé de Feriet [1, p. 22. (24)]:

$F_{1}[\alpha ; \beta, \rho ; \gamma ; 1,1]=\frac{\Gamma(\gamma) \Gamma(\gamma-\alpha-\beta-\rho)}{\Gamma(\gamma-\alpha) \Gamma(\gamma-\beta-\rho)}$

$$
\text { provided that } R(\gamma-\alpha-\beta-\rho)>0,
$$

$$
\begin{aligned}
= & \frac{\Gamma(\beta) \Gamma\left(\beta+f_{1}+f_{2}-g_{1}-g_{2}-\alpha\right)}{\Gamma(\beta-\alpha) \Gamma\left(\beta+f_{1}+f_{2}-g_{1}-g_{2}\right)} \\
& F\left[\begin{array}{c}
\alpha, \beta+f_{1}+f_{2}-g_{1}-g_{2}-\alpha ; 1 / 2 f_{1}, 1 / 2+1 / 2 f_{1} ; 1 / 2 f_{2}, 1 / 2+1 / 2 f_{2} ; 1,1 \\
1 / 2\left(\beta+f_{1}+f_{2}-g_{1}-g_{2}\right), 1 / 2\left(\beta+f_{1}+f_{2}-g_{1}-g_{2}+1\right) ; 1+f_{1} ; 1+f_{2} ;
\end{array}\right] .
\end{aligned}
$$

This completes the proof of (3) under the condition stated therein. We shall mention some of the interesting particular cases of (3).

(a) In case $a=-n$ (a positive integer) in (3), we get 


$$
F\left[\begin{array}{c}
-n, \beta+f_{1}+f_{2}+n ; 1 / 2 f_{1}, 1 / 2+1 / 2 f_{1} ; 1 / 2 f_{2}, 1 / 2+1 / 2 f_{2} ; 1,1 \\
1 / 2\left(\beta+f_{1}+f_{2}\right), 1 / 2\left(1+\beta+f_{1}+f_{2}\right) ; 1+f_{1} ; 1+f_{2} ;
\end{array}\right]
$$

In case $f_{2}=0$ in (5), it reduces to a known result of Bailey $[2,(8.2)]$.

(b) In case $f_{2}=0$ in (3), we get a new summation formula for ${ }_{4} F_{3}(1)$ :

$$
{ }_{4} F_{3}\left[\begin{array}{c}
\alpha, \beta-\alpha, 1 / 2 \rho, 1 / 2(1+\rho) ; 1 \\
1 / 2 \beta, 1 / 2(1+\beta), 1+\rho ;
\end{array}\right]=\frac{\Gamma(\beta) \Gamma(\beta-\alpha-\rho)}{\Gamma(\beta-\rho) \Gamma(\beta-\alpha)},
$$

valid for $R(\beta-\alpha-\rho)>0, R(\beta-\alpha)>$ and $R(\beta-\rho)>0$.

If $\alpha=-n$ (a positive integer) in (6), it reduces to a known result of Bailey [2].

In case $\beta=1+\alpha+\rho$ in (6), we have

$$
{ }_{3} F_{2}\left[\begin{array}{c}
\alpha, 1 / 2 \rho, 1 / 2(1+\rho) ; 1 \\
1 / 2(1+\alpha+\rho), 1 / 2(2+\alpha+\rho) ;
\end{array}\right]=\frac{\Gamma(1+\alpha+\rho)}{\Gamma(1+\alpha) \Gamma(1+\rho)},
$$

valid for $R(1+\alpha)>0, R(1+\rho)>0$ and $R(1+\alpha+\rho)>0$.

In case $\beta=1+\rho$ in (6), we have

$$
{ }_{3} F_{2}\left[\begin{array}{c}
\alpha, 1+\rho-\alpha, 1 / 2(1+\rho) ; 1 \\
1+\rho, 1 / 2(2+\rho) ;
\end{array}\right]=\frac{\Gamma(1+\rho) \Gamma(1-\alpha)}{\Gamma(1+\rho-\alpha)},
$$

valid for $R(1+\rho)>0, R(1-\alpha)>0$ and $R(1+\rho-\alpha)>0$.

\section{REFERENCES}

1. P. Appell and J. Kampé de Feriet, Fonctions hypergéométriques et hypersphériques, polynômes d'Hermite, Gauthier-Villars, Paris, 1926.

2. W. N. Bailey, Some identities involving generalised hypergeometric series, Proc. London Math. Soc. (2) 29 (1929), 503-516.

3. T. W. Chaundy, Expansions of hypergeometric functions, Quart. J. Math. Oxford Ser. 13 (1942), 159-171. MR 4, 197.

4. L. Carlitz, A Saalschützian theorem for double series, J. London Math. Soc. 38 (1963), 415-418. MR 28 \#4153.

5. - Summation of a double hypergeometric series, Matematiche (Catania) 22 (1967), 138-142. MR 35 \#5665.

6. R. N. Jain, Sum of a double hypergeometric series, Matematiche (Catania) 21 (1966), 300-301. MR 34 \#1582.

7. L. J. Slater, Generalized hypergeometric functions, Cambridge Univ. Press, Cambridge, 1966. MR 34 \#1570.

8. B. L. Sharma, Summation of a double hypergeometric series, Matematiche (Catania) 28 (1973), 1-3.

DEPARTMENT OF MA THEMATICS, UNIVERSITY OF IFE, ILE-IFE, NIGERIA 